\title{
Clinical analysis of 82 cases of carcinoma showing thymus-like differentiation of the thyroid
}

\author{
WEI GE, YONG-ZHONG YAO, GANG CHEN and YI-TAO DING \\ Department of General Surgery, Nanjing Drum Tower Hospital, \\ The Affiliated Hospital of Nanjing University Medical School, Nanjing, Jiangsu 210008, P.R. China
}

Received December 5, 2014; Accepted November 6, 2015

DOI: $10.3892 / \mathrm{ol} .2015 .4055$

\begin{abstract}
The aim of the present study was to summarize the clinical characteristics, diagnosis, treatment and prognosis of carcinoma showing thymus-like differentiation of the thyroid (CASTLE). A search of the relevant literature was conducted, which identified 82 cases of CASTLE reported to date. A review of the clinical and auxiliary examination data, treatment, pathological findings and follow-up of these cases was performed. The clinical manifestations of CASTLE varied and did not demonstrate specificity. During ultrasound examination, CASTLE was described as a solid and hypoechoic mass, and the echo was typically observed to be heterogeneous. In computed tomography (CT), CASTLE was demonstrated to be a well-defined, soft tissue density mass without calcification, which appeared enhanced following administration of contrast medium. In emission CT, CASTLE was revealed as a 'cold nodule'. A total of 78 patients underwent radical surgery, and 12 patients experienced recurrence. Lymph node metastasis did not promote recurrence, and postoperative radiation was not able to reduce the recurrence rate $(\mathrm{P}=0.144$ and 1.000 , respectively). The median follow-up time was 14 months (range, 1-312 months). Typical immunohistochemical examinations demonstrated the specimens to be positive for cluster of differentiation (CD)5, CD117, cytokeratin 19, epithelial membrane antigen and tumor protein 63, and negative for calcitonin, thyroglobulin and thyroid transcription factor-1. Thyroid CASTLE is a rare malignancy whose exact diagnosis relies on pathological examination, particularly immunohistochemistry, since preoperative examinations are usually unable to provide an exact diagnosis. Radical resection is the primary treatment for CASTLE, which presents favorable prognosis.
\end{abstract}

Correspondence to: Dr Gang Chen, Department of General Surgery, Nanjing Drum Tower Hospital, The Affiliated Hospital of Nanjing University Medical School, 321 Zhongshan Road, Nanjing, Jiangsu 210008, P.R. China

E-mail: 331812195@qq.com

Key words: carcinoma showing thymus-like differentiation of the thyroid, thyroid, thymus-like, cluster of differentiation 5, cluster of differentiation 117

\section{Introduction}

Carcinoma showing thymus-like differentiation of the thyroid (CASTLE) is a rare intrathyroidal neoplasm, which may arise from ectopic thymus tissue or branchial pouch remnants (1). Chan and Rosai (2) classified the clinicopathological features of CASTLE into four types: i) Ectopic hamartomatous thymoma; ii) ectopic cervical thymoma; iii) spindle epithelial tumor with thymic-like differentiation; iv) and CASTLE. At present, CASTLE is considered an independent clinicopathological entity of thyroid tumors, according to the World Health Organization classification of tumors of endocrine organs (3). To the best of our knowledge, solely 3 patients have presented with CASTLE at Nanjing Drum Tower Hospital (The Affiliated Hospital of Nanjing University Medical School; Nanjing, China) to date. Due to the rarity of CASTLE, there is currently no unified standard method of diagnosis or therapy (1). In 2013, Huang et al (4) summarized 45 cases of CASTLE identified in China. To fully understand this disease, a search of the relevant literature in Pubmed and Chinese databases was performed in the present study, which identified a total of 82 cases of CASTLE reported to date. These cases are summarized in the present study, and recommendations for the diagnosis and treatment of CASTLE are suggested.

\section{Materials and methods}

Database search. Electronic searches of the PubMed (www. ncbi.nlm.nih.gov/pubmed), China National Knowledge Infrastructure (www.global.cnki.net/kns50/single_index.aspx) and Ten Thousand databases (http://g.wanfangdata.com.cn) were performed using the following key words: Thyroid, thymus and carcinoma. A total of 82 patients with CASTLE were identified, and their clinical characteristics, diagnosis, treatment, pathology and follow-up were reviewed.

Review of preoperative examinations. The results of preoperative examinations, including ultrasound $(n=57)$, computed tomography (CT; $n=27)$, emission CT (ECT; $n=10)$, thyroid function $(n=52)$ and fine-needle aspiration cytology $(n=24)$, were reviewed. Histopathological examination of surgical specimens was performed using standard hematoxylin and eosin staining (H\&E), alongside specific immunohistochemical techniques. The primary immunohistochemical markers 
were cluster of differentiation (CD)5, CD117, cytokeratin (CK), CK19, CK AE1/AE3, tumor protein (TP)63, carcinoembryonic antigen (CEA), synaptophysin, B-cell lymphoma 2, epithelial membrane antigen (EMA), chromogranin A, calcitonin (CT), thyroglobulin (TG) and thyroid transcription factor-1 (TTF-1). All images are of a single patient and are representative of all patients analyzed.

Statistical analysis. The $\chi^{2}$ test was used to evaluate the effects of lymph node metastasis and radiotherapy on recurrence. $\mathrm{P}<0.05$ was considered to indicate a statistically significant difference. All P-values were two-sided. All statistical calculations were performed using SPSS software version 19.0 (IBM SPSS, Armonk, NY, USA).

\section{Results}

Baseline characteristics demonstrated wide variations. Of the 82 cases reviewed in the present study, 59 patients were Chinese, 11 were Japanese, 9 were American, 1 was Italian, 1 was Korean and 1 was Portuguese. A total of 37 patients were males and 45 were females. The male:female ratio was 1:1.22. The average patient age was $48.8 \pm 12.8$ years (range, 25-79 years). The patients presented with a range of symptoms, including a neck tumor in 40 cases (48.78\%), hoarseness in 13 patients $(15.85 \%)$, dyspnea in 8 patients $(9.76 \%)$, cough in 3 patients $(3.66 \%)$, neck pain in 3 patients $(3.66 \%)$ and dysphagia in 1 patient (1.22\%). For 14 patients $(17.07 \%)$, the symptoms were unknown. A total of 34 CASTLE tumors were located in the left lobe of the thyroid, 44 were in the right lobe and 3 were bilateral. In 1 patient, the location of the tumor was unknown. The location of the tumor was more specifically described in 50 cases, of which, 47 presented the tumor in the lower part of the thyroid and 3 in the upper part. The baseline characteristics of the patients are summarized in Table I.

Preoperative examinations varied from patient to patient. In the present study, descriptions of ultrasound examination were recorded for 57 patients, whose CASTLE was typically described as a solid and hypoechoic mass with heterogeneous echo (Fig. 1). Results of ECT in 10 patients were acquired in the present study, and all tumors were described as 'cold nodule'. CT examination in 27 patients revealed that CASTLE was a well-defined, soft tissue density mass without calcification, and the mass appeared enhanced following administration of contrast medium. A total of 52 patients underwent a test of thyroid function. Of these, 51 cases were normal and 1 exhibited hyperthyroidism. A total of 24 patients underwent fine-needle aspiration cytology. Of these, 2 patients were diagnosed with CASTLE, 17 cases were demonstrated to be malignant tumors, 1 case was hyperplasia and the remaining 4 cases were negative.

Treatment and follow-up demonstrated wide variations across patients. Of the 81 patients who had undergone surgery, lymph node metastasis was observed in 22 patients, and distant metastasis to the lung was identified in 2 patients. Postoperative radiation was administered as an adjuvant therapy in 29 patients, while 4 patients received chemotherapy in addition to radiation. The median follow-up time was 14 months
Table I. Clinical characteristics of 82 patients with carcinoma showing thymus-like differentiation of the thyroid.

\begin{tabular}{|c|c|c|}
\hline Parameter & Number of patients & Percentage \\
\hline \multicolumn{3}{|l|}{ Country } \\
\hline China & 59 & 71.95 \\
\hline Japan & 11 & 13.41 \\
\hline America & 9 & 10.98 \\
\hline Italy & 1 & 1.22 \\
\hline Korea & 1 & 1.22 \\
\hline Portugal & 1 & 1.22 \\
\hline \multicolumn{3}{|l|}{ Age, years } \\
\hline$<40$ & 23 & 28.05 \\
\hline $40-60$ & 40 & 48.78 \\
\hline$>60$ & 19 & 23.17 \\
\hline \multicolumn{3}{|l|}{ Gender } \\
\hline Male & 37 & 45.12 \\
\hline Female & 45 & 54.88 \\
\hline \multicolumn{3}{|l|}{ Symptom } \\
\hline Neck tumor & 40 & 48.78 \\
\hline Hoarseness & 13 & 15.85 \\
\hline Dyspnea & 8 & 9.76 \\
\hline Cough & 3 & 3.66 \\
\hline Neck pain & 3 & 3.66 \\
\hline Dysphagia & 1 & 1.22 \\
\hline Unknown & 14 & 17.07 \\
\hline \multicolumn{3}{|l|}{ Thyroid location } \\
\hline Left lobe & 34 & 41.46 \\
\hline Right lobe & 44 & 53.66 \\
\hline Bilateral lobe & 3 & 3.66 \\
\hline Unknown & 1 & 1.22 \\
\hline
\end{tabular}

(range, 1-312 months), and no follow-up period was reported for 15 patients. Of the 78 patients who had undergone radical surgery, 12 experienced recurrence, with a median time to recurrence of 11 months (range, 1-144 months; Table II). The effect of lymph node metastasis and radiotherapy on recurrence was subsequently evaluated by $\chi^{2}$ test using SPSS software version 19.0 (Table III).

Pathological and immunohistochemical examinations confirmed positivity for certain markers. Microscopic examination revealed the presence of confluent nests and lobules of various glands with a few lymphocytes and plasma cell infiltration (Fig. 2). The immunohistochemical results are summarized in Table IV. Typical immunohistochemical examinations demonstrated positivity for CD5, CD117, CK19, EMA and TP63, and negativity for CT, TG and TTF-1 (Fig. 3).

\section{Discussion}

CASTLE is a rare, indolent and slow-growing malignant neoplasm of the thyroid gland, which was initially reported by Miyauchi et al in 1985 (5). According to the relevant literature, 
Table II. Follow-up of 12 patients exhibiting carcinoma showing thymus-like differentiation of the thyroid who experienced recurrence.

\begin{tabular}{|c|c|c|c|c|}
\hline Authors & Time until recurrence ${ }^{a}$, months & Treatment & Follow-up time & Dutcome \\
\hline Liu et al (6) & 144 & Surgery & 27 & NED \\
\hline Liu et al (6) & 22 & Surgery & 44 & NED \\
\hline Bai et al (7) & 22 & Surgery and radiotherapy & 50 & NED \\
\hline Pan et al (8) & 3 & Surgery, radiotherapy and chemotherapy & 12 & NED \\
\hline Luo et al (9) & 24 & Surgery, radiotherapy and chemotherapy & 6 & NED \\
\hline Wu et al (10) & 1 & Radiotherapy & 25 & NED \\
\hline Da et al (11) & 6 & Surgery & 108 & NED \\
\hline Da et al (11) & 6 & Surgery & 132 & NED \\
\hline Da et al (11) & 3 & Unknown & 36 & SD \\
\hline Reimann et al (13) & 72 & Radiotherapy & 96 & SD \\
\hline Reimann et al (13) & 36 & Surgery & 96 & NED \\
\hline Tsutsui et al (12) & 16 & Surgery & 34 & NED \\
\hline
\end{tabular}

${ }^{a}$ Following surgery. ${ }^{b}$ Following treatment. NED, no evidence of disease; SD, succumbed to disease.

Table III. Effect of lymph node metastasis and radiotherapy on recurrence.

\begin{tabular}{lccc}
\hline Parameter & Recurrence & No recurrence & $\chi^{2}$ value \\
\hline Lymph node metastasis & 6 & 13 & 1.482 \\
Present & 3 & 22 & 0.144 \\
Absent & & 25 & 0.000 \\
Radiotherapy & 4 & 16 & 1.000 \\
Present & 3 & & \\
Absent & & & \\
\hline
\end{tabular}

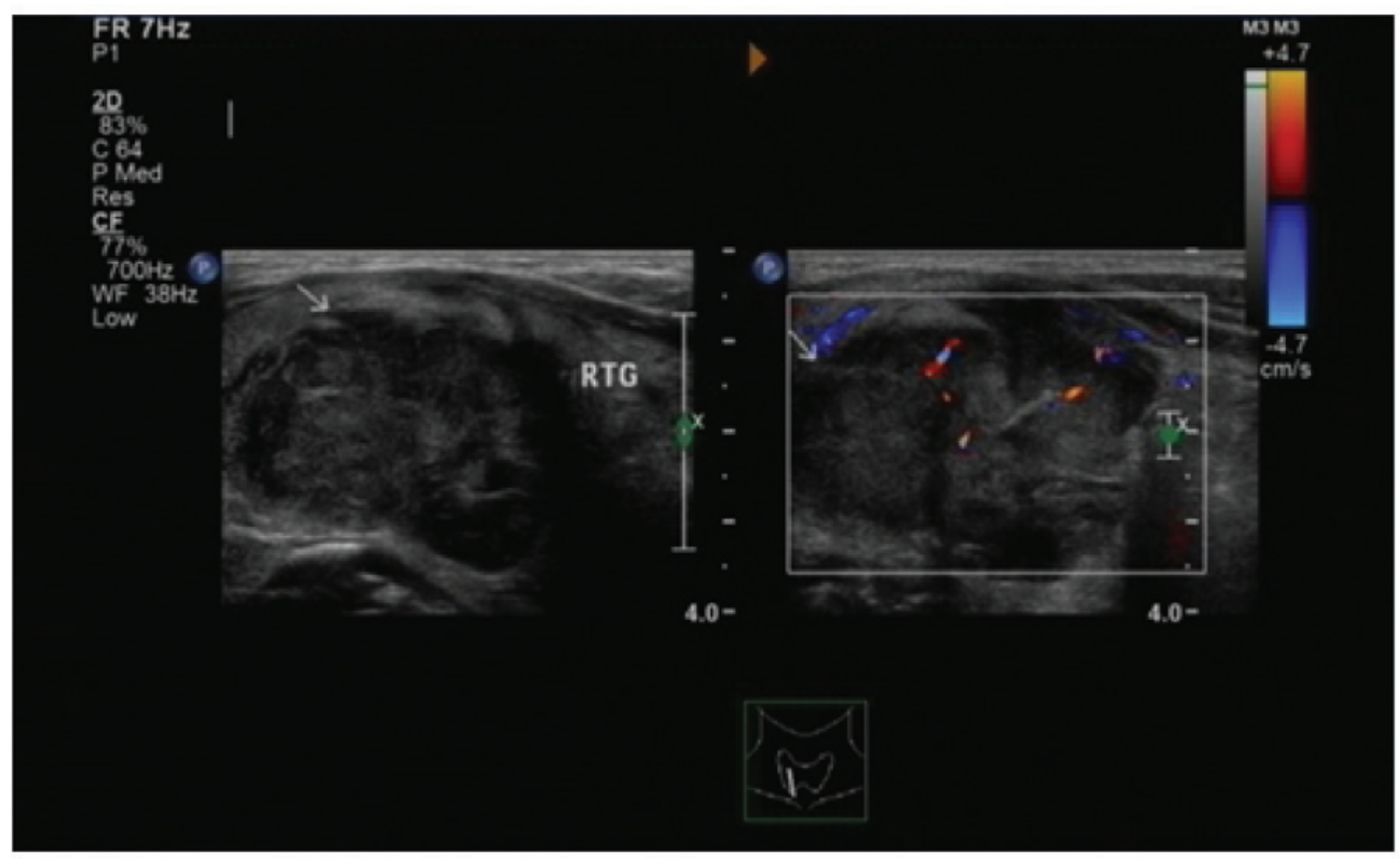

Figure 1. Ultrasound revealed a mass of $3.8 \times 2.7 \mathrm{~cm}$ located in the right lobe of the thyroid. The border between the tumor and the thyroid gland, and between the tumor and the surrounding fat, was irregular. The tumor was solid and displayed a heterogeneous hypoechoic pattern. 
Table IV. Immunohistochemical results of 82 cases of carcinoma showing thymus-like differentiation of the thyroid.

\begin{tabular}{lccr}
\hline Immunological marker & Number of cases studied & Number of positive cases & Percentage \\
\hline CD5 & 63 & 63 & 100.00 \\
CK & 19 & 10 & 52.63 \\
CD117 & 37 & 36 & 97.30 \\
CK19 & 14 & 14 & 100.00 \\
Tumor protein 63 & 32 & 32 & 100.00 \\
Carcinoembryonic antigen & 25 & 16 & 64.00 \\
Synaptophysin & 19 & 5 & 26.32 \\
CK AE1/AE3 & 11 & 11 & 100.00 \\
B-cell lymphoma 2 & 14 & 14 & 100.00 \\
Epithelial membrane antigen & 7 & 5 & 71.43 \\
Chromogranin A & 9 & 2 & 22.22 \\
Thyroglobulin & 55 & 0 & 0.00 \\
Calcitonin & 35 & 0 & 0.00 \\
Thyroid transcription factor-1 & 51 & 0 & 0.00 \\
\hline
\end{tabular}

$\mathrm{CD}$, cluster of differentiation; CK, cytokeratin.
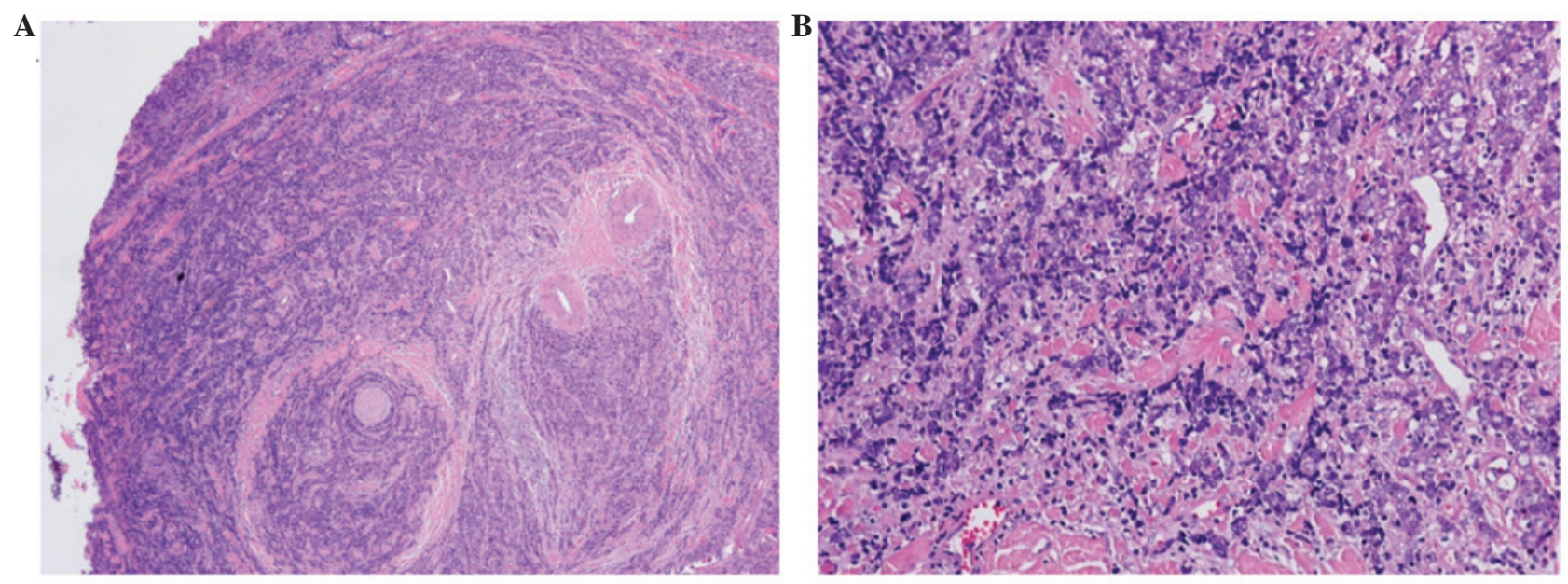

Figure 2. Microscopic examination revealed confluent nests and lobules of various glands with a few lymphocytes and plasma cell infiltration. (A) Hematoxylin and eosin staining; magnification, (A) x40 and (B) x200.

and to the best of our knowledge, 82 cases of CASTLE have been reported thus far, primarily in the form of case reports. The present study summarized the clinical characteristics of all the cases of CASTLE published to date in the relevant literature. The male:female ratio was 1:1.22, and the average patient age was $48.8 \pm 12.8$ years (range, $25-79$ years). The clinical manifestations of CASTLE varied across the patients, and were not specific. The majority of previous reports have described CASTLE tumors involving predominantly the lower part of the thyroid, which may be due to CASTLE originating from ectopic thymic tissue or branchial pouch remnants in or adjacent to the thyroid $(2,14)$.

The preoperative diagnosis of thyroid tumors relies on ultrasound, ECT, CT, thyroid function and fine-needle aspiration cytology (1,6-11). In the present study, ultrasound imaging of CASTLE identified a solid and hypoechoic mass, whose echo was typically heterogeneous. Yamamoto et al (15) studied the sonographic findings in three cases of CASTLE and concluded that CASTLE was solid and hypoechoic, with a heterogeneous echo pattern and without cystic components or calcifications, which is in agreement with the results of the present study. CASTLE was previously described as 'cold nodule' in ECT, suggesting that it was a malignancy (1). In CT, CASTLE was revealed as a well-defined, soft tissue density mass without calcification, and the mass appeared enhanced following administration of contrast medium $(16,17)$. Tests of thyroid function were normal in almost all the CASTLE cases analyzed, suggesting that CASTLE did not affect the thyroid function (1). A total of 24 patients underwent fine-needle aspiration cytology. Of these, 2 were diagnosed with CASTLE, thus indicating that fine-needle aspiration cytology contributed to the primary diagnosis of CASTLE, while its exact diagnosis depended on pathological and immunohistochemical examination. However, due to the false-negative rates 
A

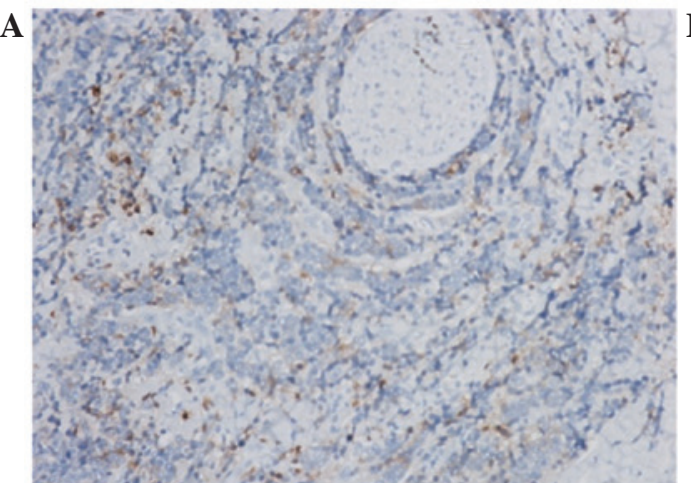

C

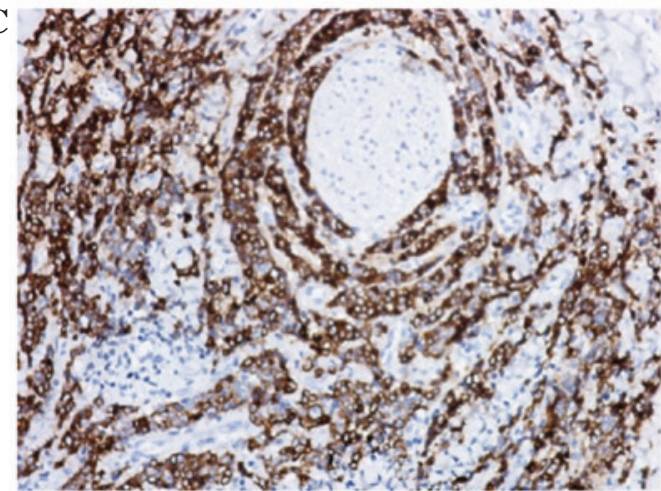

$\mathbf{E}$
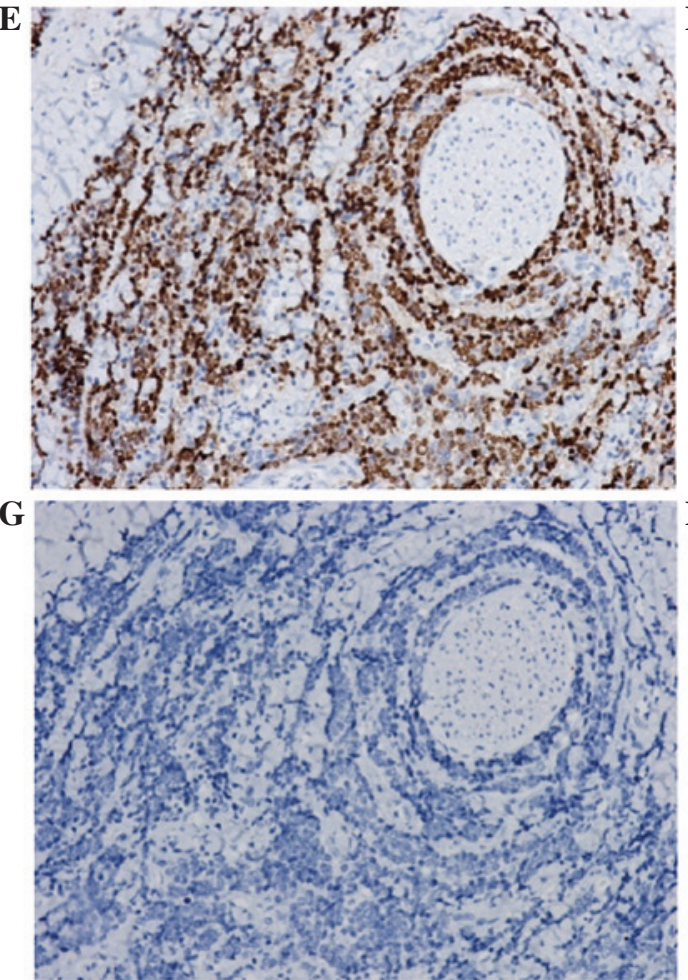

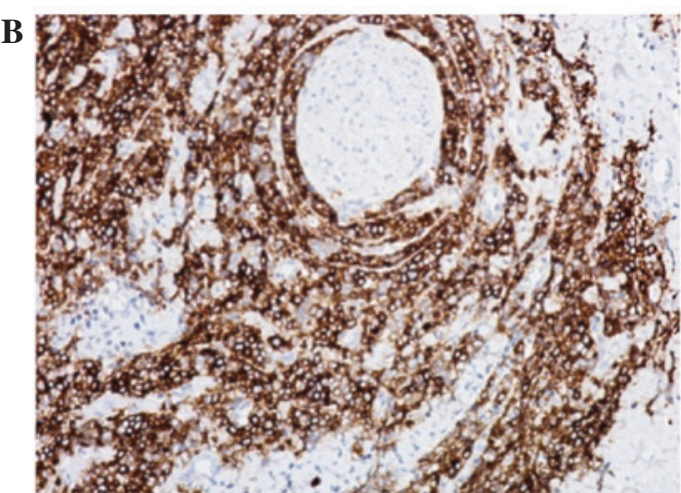

D
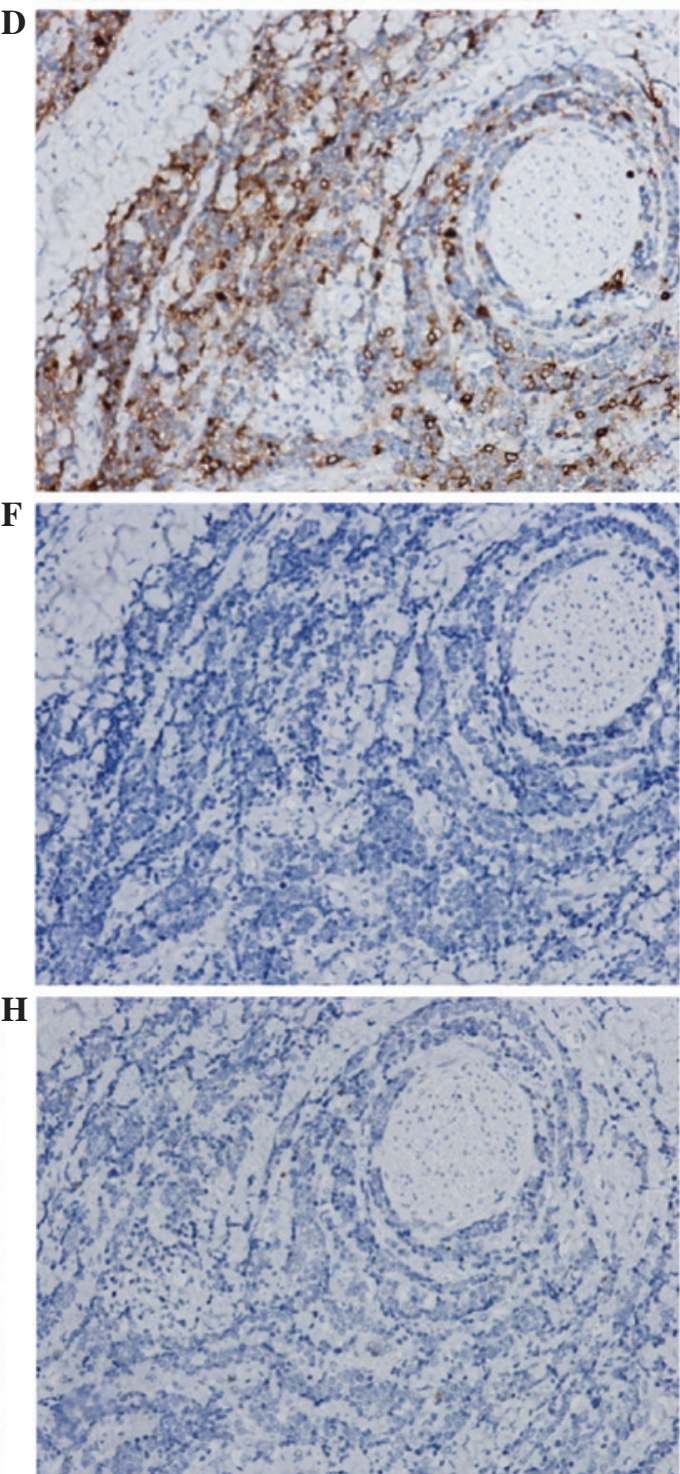

Figure 3. Immunohistochemical examination demonstrated the specimens to be positive for (A) CD5, (B) CD117, (C) cytokeratin 19, (D) epithelial membrane antigen and (E) tumor protein 63, and negative for $(\mathrm{F})$ calcitonin, $(\mathrm{G})$ thyroglobulin and $(\mathrm{H})$ thyroid transcription factor-1. CD, cluster of differentiation.

exhibited by fine-needle aspiration cytology, the results should be interpreted with caution, and the examination repeated if required (18).

The preoperative diagnosis of CASTLE is difficult, and conclusive diagnosis often relies on pathological examination, particularly immunohistochemistry (1). A number of studies have indicated that CD5 may be a useful marker for the diagnosis of CASTLE, as it is a marker for carcinoma of thymic origin $(19,20)$. Similar to thymic carcinoma, CASTLE is immunohistochemically positive for CD5, but negative for CT, TG and TTF-1 (19). Ito et al (21) reported a sensitivity and specificity of 82 and $100 \%$, respectively, for CD5 positivity for the diagnosis of CASTLE. In the present study, 63 patients underwent CD5 testing and all demonstrated positivity for this marker, while all the cases tested demonstrated negativity for CT, TG and TTF-1. However, 
since a lack of CD5 expression does not completely rule out CASTLE, the final diagnosis was based on H\&E findings. A previous study reported that expression of high molecular weight CK, CEA and TP63 in CASTLE provided evidence of the thymic origin of the tumor, and were useful diagnostic markers for distinguishing thyroid CASTLE from other thyroid neoplasms (13). In the present study, the positive rates for CK19, CEA and TP63 were 100.00, 64.00 and 100.00\%, respectively. Reimann et al (13) reported that the majority of nuclei stained positively for TP63 in 11/11 thyroid solid cell nests, 7/7 CASTLEs, 10/10 thymic carcinomas and $23 / 23$ thymomas. In the present study, TP63 was examined in 32 patients and all cases proved to be positive. In addition, CD117 may be a useful maker for distinguishing CASTLE from other thyroid tumors, since the results of the present study demonstrated a positive rate for CD117 expression of $97.30 \%$.

In the present study, 81 patients underwent surgery. Lymph node metastasis was observed in 22 patients, however, only 19 patients with lymph node metastasis completed follow-up. Postoperative radiation was administered as an adjuvant therapy to 28 patients, and 4 patients received chemotherapy in addition to radiation. Of the 78 patients who had undergone radical surgery, 12 experienced recurrence. The present study evaluated the effects of lymph node metastasis and radiotherapy on recurrence. The results demonstrated that lymph node metastasis did not promote recurrence following radical surgery $(\mathrm{P}=0.144)$, and postoperative radiation was not able to reduce the recurrence rate $(\mathrm{P}=1.000)$. By contrast, Roka et al (22) reported that node-negative CASTLE presented a low risk of recurrence, and that curative surgery of the tumor with resection of the adjacent organs involved in addition to systematic dissection of the lymph nodes, followed by radiation therapy, was able to prevent locoregional recurrence. Therefore, the findings of the present study require further confirmation, due to the limited sample size of the study.

The prognosis of CASTLE is relatively positive (12). In the present study, the recurrence rate was $18.57 \%$ (13/70 patients). During follow-up, 4 patients were reported to succumb to disease, and the longest follow-up time of these 4 patients was 26 years.

In conclusion, thyroid CASTLE is a rare malignancy of the thyroid gland that may display various manifestations. Preoperative examinations, including CT, ECT and ultrasound, may not be capable of establishing an exact diagnosis, which typically requires pathological examination, particularly immunohistochemical results. Radical resection is the primary treatment for CASTLE, and the prognosis for this disease is favorable following surgery. Postoperative radiation appears to be unable to reduce the recurrence rate in patients with CASTLE.

\section{References}

1. Liu Z, Teng XY, Sun DX, Xu WX and Sun SL: Clinical analysis of thyroid carcinoma showing thymus-like differentiation: Report of 8 cases. Int Surg 98: 95-100, 2013.

2. Chan JK and Rosai J: Tumors of the neck showing thymic or related branchial pouch differentiation: A unifying concept. Hum Pathol 22: 349-367, 1991.
3. DeLellis RA, Lloyd RV, Heitz PU and Eng C (eds): Parathyroid carcinoma. In: World Health Organization Classification of Tumours. Pathology and Genetics of Tumors of Endocrine Organs. Vol 96. IARC Press, Lyon, pp124-127, 2004.

4. Huang C, Wang L, Wang Y, Yang X and Li Q: Carcinoma showing thymus-like differentiation of the thyroid (CASTLE). Pathol Res Pract 209: 662-665, 2013.

5. Miyauchi A, Kuma K, Matsuzuka F, Matsubayashi S, Kobayashi A, Tamai $\mathrm{H}$ and Katayama S: Intrathyroidal epithelial thymoma: An entity distinct from squamous cell carcinoma of the thyroid. World J Surg 9: 128-135, 1985.

6. Liu X, Hadeti B, Zhang W and Wang J: Thyroid carcinoma showing thymus-like differentiation: A clinicopathologic study of 8 cases. Zhonghua Bing Li Xue Za Zhi 40: 89-93, 2011 (In Chinese)

7. Bai T, Zhang YF, Wu HY and Bian A: Cytological diagnosis of carcinoma shwoing thymus-like differentiation of the thyroid gland. Acta Acad Med Xuzhou 12: 802-805, 2010 (In Chinese).

8. Pan YL, Xie Y, Pu WL, Luo ZM, Yuan CY and Cai PR: Carcinoma showing thymus-like element of the thyroid: A clinicopathological observation. J Diagn Pathol 5: 373-376, 2007 (In Chinese).

9. Luo J, Ni XH, Zhang G, et al: Clinicopathologic features of carcinoma showing thymus-like differentiation in the neck. J Oncol 11: 906-909, 2008 (In Chinese).

10. Wu HP, Zhang ZP and Pan YL: Thyroid carcinoma showing thymus-like differentiation: A case report. J Diagn Concepts Pract 6: 288, 2007 (In Chinese).

11. Da J, Shi H and Lu J: Thyroid squamous-cell carcinoma showing thymus-like element (CASTLE): A report of eight cases. Zhonghua Zhong Liu Za Zhi 21: 303-304, 1999 (In Chinese).

12. Tsutsui H, Hoshi M, Kubota M, Suzuki A, Nakamura N, Usuda J, Shibuya H, Miyajima K, Ohira T, Ito K and Ikeda N: Management of thyroid carcinoma showing thymus-like differentiation (CASTLE) invading the trachea. Surg Today 43: 1261-1268, 2013.

13. Reimann JD, Dorfman DM and Nosé V: Carcinoma showing thymus-like differentiation of the thyroid (CASTLE): A comparative study: Evidence of thymic differentiation and solid cell nest origin. Am J Surg Pathol 30: 994-1001, 2006.

14. Yoneda K, Matsui O, Kobayashi T, Gabata T, Minato H and Hirokawa M: CT and MRI findings of carcinoma showing thymus-like differentiation. Radiat Med 23: 451-455, 2005.

15. Yamamoto Y, Yamada K, Motoi N, Fujiwara Y, Toda K, Sugitani I and Kohno A: Sonographic findings in three cases of carcinoma showing thymus-like differentiation. J Clin Ultrasound 41: 574-578, 2013.

16. Sun T, Wang Z, Wang J, Wu Y, Li D and Ying H: Outcome of radical resection and postoperative radiotherapy for thyroid carcinoma showing thymus-like differentiation. World J Surg 35: 1840-1846, 2011

17. Kusada N, Hara Y, Kobayashi S, Weihua T, Nakamura Y, Kakudo K and Yuasa H: A case of aggressive carcinoma showing thymus-like differentiation with distant metastases. Thyroid 15: 1383-1388, 2005

18. Chang S, Joo M and Kim H: Cytologic findings of thyroid carcinoma showing thymus-like differentiation: A case report. Korean J Pathol 46: 302-305, 2012.

19. Kakudo K, Bai Y, Ozaki T, Homma K, Ito Y and Miyauchi A: Intrathyroid epithelial thymoma (ITET) and carcinoma showing thymus-like differentiation (CASTLE): CD5-positive neoplasms mimicking squamous cell carcinoma of the thyroid. Histol Histopathol 28: 543-556, 2013.

20. Dorfman DM, Shahsafaei A and Miyauchi A: Intrathyroidal epithelial thymoma (ITET)/carcinoma showing thymus-like differentiation (CASTLE) exhibits CD5 immunoreactivity: New evidence for thymic differentiation. Histopathology 32: 104-109, 1998.

21. Ito Y, Miyauchi A, Nakamura Y, Miya A, Kobayashi K and Kakudo K: Clinicopathologic significance of intrathyroidal epithelial thymoma/carcinoma showing thymus-like differentiation: A collaborative study with Member Institutes of The Japanese Society of Thyroid Surgery. Am J Clin Pathol 127: 230-236, 2007

22. Roka S, Kornek G, Schüller J, Ortmann E, Feichtinger J and Armbruster C: Carcinoma showing thymic-like elements - a rare malignancy of the thyroid gland. Br J Surg 91: 142-145, 2004. 